\title{
Germanica
}

unifiée

\section{Wie es leuchtet. Léonce et Léna de l'unification allemande}

Thomas Brussig, Wie es leuchtet, Leonce und Lena der deutschen Vereinigung

\section{Marie-Hélène Quéval}

\section{OpenEdition}

\section{Journals}

Édition électronique

URL : http://journals.openedition.org/germanica/607

DOI : $10.4000 /$ germanica.607

ISSN : 2107-0784

Éditeur

Université de Lille

Édition imprimée

Date de publication : 1 juin 2009

Pagination : $33-50$

ISBN : 978-2-913857-23-0

ISSN : 0984-2632

Référence électronique

Marie-Hélène Quéval, «Wie es leuchtet. Léonce et Léna de l'unification allemande », Germanica [En

ligne], 44 | 2009, mis en ligne le 01 juin 2011, consulté le 06 octobre 2020. URL : http://

journals.openedition.org/germanica/607 ; DOI : https://doi.org/10.4000/germanica.607

Ce document a été généré automatiquement le 6 octobre 2020.

(c) Tous droits réservés 


\title{
Wie es leuchtet. Léonce et Léna de l'unification allemande
}

Thomas Brussig, Wie es leuchtet, Leonce und Lena der deutschen Vereinigung

\author{
Marie-Hélène Quéval
}

1 Nombreuses sont les voix nostalgiques qui, sans regretter le régime du SED en RDA, jettent un regard mélancolique sur un passé définitivement englouti par le raz de marée de la Réunification allemande. Beaucoup en effet ont ressenti le tournant de 1989 comme un cataclysme naturel semblable à celui du déluge biblique qui avait anéanti l'humanité tout entière. Jens Sparschuh déjà compare la fin de la RDA à celle de Vineta, tandis que, dans son roman Wie es leuchtet (2004), Brussig confère une dimension mythique à l'événement en lui associant la crue extraordinaire de l'Elbe, ce qui d'ailleurs situe l'action du roman en 2002.

2 Au-delà de la tradition judéo-chrétienne, la Mésopotamie et la Grèce antique ont conservé le souvenir d'une catastrophe climatique entraînant la disparition de l'humanité et de sa civilisation. Sans même faire référence au mythe de Deucalion, Platon imagine, dans ses deux dialogues Timée et Timias, la légende de l'Atlantide précipitée au fond de l'océan. Mais si, chez Platon, le cataclysme submerge une cité bienheureuse, une culture très avancée et fort ingénieuse, les débordements de l'Elbe ont au contraire mis fin à une civilisation présentée comme perverse et criminelle. On aurait donc tort de comparer la RDA disparue à la florissante Atlantide. L'Arche de Noé imaginée par Brussig, loin de sauver les survivants vertueux d'un monde condamné pour sa corruption, prend ici l'allure ridicule de plusieurs pianos à queue et droits entassés les uns sur les autres comme les musiciens de Brême ! L'image donne le ton : la fin de la RDA est un événement grotesque conforme à la réalité politique de ce pays ${ }^{1}$. L'incipit du roman fait de la spectaculaire inondation d'août 2002 l'événement fondateur du nouveau monde auquel les citoyens est-allemands se voient confrontés sans y avoir été préparés. En une seule nuit, les archives du photographe, et avec elles la mémoire nationale, disparaissent dans le fleuve en colère. Des clichés du narrateur documentant la Révolution de 1989, die Wende, il ne reste plus que des images floues, 
effacées par l'humidité, des ombres chancelantes, sans contours... Le "tournant" historique aurait-il déjà perdu de son importance ou serait-il impossible à représenter?

Brussig ne peut pas oublier ce moment magique où l'être se transforme, l'expérience heureuse de la résurrection lorsque tout bascule, disparaît, meurt et renaît... comme si la nouvelle identité ne pouvait surgir que de la mort du monde ancien. Le Stirb und werde goethéen qui exhorte à sortir de soi-même, à tuer en soi le passé, est en effet la condition nécessaire pour pouvoir se continuer en rejetant la chrysalide, pour laisser s'épanouir le beau papillon, lui permettre de s'arracher à la glaise et de prendre son envol.

$\mathrm{M}^{\mathrm{me}}$ Warthe jeta une poignée de terre dans la tombe, et en levant les yeux, elle aperçut un papillon qui, entrainé par son geste, prit son envol'2.

\section{La théorie du Chaos}

\section{L'effet du papillon}

4 Le comportement imprévisible de l'Elbe aurait-il fait surgir par association d'idées les réflexions de Lorenz sur les phénomènes météorologiques désordonnés, imprévisibles donc, qualifiés à juste titre de "chaotiques»? Peu importe! Lorsque Léna applique à l'unification allemande la «théorie du chaos ", elle fait certes allusion à Edward Norton Lorenz $\mathrm{z}^{3}$ (1917-2008), mais aussi à une vogue des années 1970. D’abord formulée par James Clerk Maxwell (1831-1879), puis par Henri Poincaré (1854-1912), cette théorie fut reprise par le météorologue qui avait remarqué que le plus petit changement dans son programme météorologique pouvait entraîner des catastrophes climatiques dans des régions très éloignées, observation qu'il avait exprimée par la métaphore de «l'effet du papillon» dont le moindre battement d'aile pourrait au fil des années provoquer un typhon à l'autre bout du monde. Si Lorenz voulait contester le déterminisme absolu du modèle newtonien, Léna se contente d'une formule plus simple selon laquelle le moindre changement dans le comportement d'une personne pourrait engendrer d'énormes conséquences dans son existence.

Tout est chaos ! Elle jeta ses mains en l'air, pour montrer ce qu'elle voulait dire. Elle était encore essoufflée et dut reprendre son souffle à plusieurs reprises, avant de pouvoir continuer. «La théorie du Chaos dit que le battement d'ailes d'un papillon en Thaillande peut mettre en mouvement une chose qui provoquera un typhon aux USA. » [...] Voilà ce qui me rend heureuse ! Car cela signifie que le maléfice ici peut prendre fin. [...] Partout les hasards nous guettent. Ce que nous croyons être l'état du monde est quelque chose de sensible, d'éphémère. Ça commence toujours comme une gouttelette d'eau. Ou comme le battement d'ailes d'un papillon. Voilà comment je le sens, comment je sens le monde ${ }^{4}$.

5 Appliquée aux mouvements protestataires de 1989, cela signifie que la chute du Mur n'est pas le résultat d'actions concertées, d'un mouvement organisé, mais celui de mille changements imperceptibles dans le comportement des citoyens de la RDA.

Un écrivain quitte le parti. Un directeur divorce. Un sportif de haut niveau jusquelà toujours docile donne d'autres interviews. Un professeur fait du yoga. Un vétérinaire devient végétarien. Un étudiant en journalisme met fin à son abonnement au journal. Un concierge cesse de fumer. Un professeur de piano suit un cours d'autodéfense. Une masseuse fait du patin à roulettes. Tous font ce qu'ils auraient dû faire depuis longtemps. Le réseau des vieilles habitudes et servitudes, d'indolence, d'indifférence et d'impuissance se distend. Bientôt il craquerait complètement ${ }^{5}$. 

avait appelé « chaosmose » la stase existentielle, pour expliquer la psychose comme un moment de création de l'être dans l'expérience de la catastrophe considérée comme une mort. Compromis par la répétition, l'être en soi s'imposerait selon Guattari comme un "continuum intensif» et un monde n'existerait que s'il contient un foyer «chaosmique » de décomposition à partir duquel s'incarnent les prises de positions subjectives. Il existerait alors une oscillation infinie entre un mouvement de «grasping chaosmique » et le déploiement incessant des coordonnées du monde. Pour Guattari, « la psychose hante ainsi non seulement la névrose et la perversion, mais aussi toutes les formes de la normalité ». La psychose, en réconciliant le chaos et la complexité, serait ainsi « une hypnose du réel ». Or, pour certains psychanalystes, seule l'hypnose permet un accès aisé, rapide et large à l'inconscient. Guattari qualifie de «chaotique l'homogénèse des référents ontologiques et, à travers elle, celle, latente, des autres modalités de subjectivation ». À la racine du monde se trouverait une forme de mise à mal chaotique.

Dans une perspective psychanalytique, la chute du Mur serait donc cet événement unique, marquant le destin de chacun des personnages comme par un

"processus d'homogénèse ontologique », mettant l'être à jour. Le sentiment de la catastrophe, la conscience de la fin d'un monde s'allie au sentiment de la rédemption, de la renaissance. Car c'est en passant par cette prise de terre chaotique, cette oscillation périlleuse, qu'autre chose devient possible, que des bifurcations ontologiques et des coefficients de créativité processuelle peuvent émerger ${ }^{6}$.

Si le schizophrène n'est pas vraiment, aux yeux de Félix Guattari, le « héros » des temps postmodernes, son mode de fonctionnement correspondrait en revanche parfaitement à la « condition postmoderne ». Brussig, sans avoir lu l'essai du psychanalyste français, renoue avec ses thèses, elles aussi très en vogue dans le Prenzlauer Berg des années 1980. Et c'est dans cette mouvance que s'intègrent les psychoses de Léna et de son grand frère, tous deux incapables de vivre sainement leur sexualité, ou encore la mégalomanie du journaliste occidental fermé au monde et obnubilé par sa propre personne.

\section{La schizophrénie d'un peuple}

9 Comme Jeanne d'Arc, Léna est une jeune pucelle, tandis que son "grand frère ", son double, est paralysé par l'expérience trouble de l'hypnose. Aucun des deux n'est capable d'accepter sa libido et, avec elle, la vie. Déjà Le Complexe de Klaus ${ }^{7}$ avait repris les thèses d'Alice Miller sur la pédagogie noire et la pédagogie blanche. Wilhelm Reich, qui a exercé une profonde influence sur la jeunesse dans les années 1970, avait très tôt établi le lien entre la répression de la sexualité et la dictature national-socialiste. Ici, la frigidité de Léna et l'impuissance de son meilleur ami servent de paradigme à la psychose de tout un peuple. Privé de passé et d'avenir, le citoyen de RDA se trouve dans l'état de déréliction décrit par Guattari. Les adultes éternellement infantilisés sont incapables de comprendre la complexité du monde, de se ressaisir, de se reconstruire, d'échapper à la pétrification et à la déterritorialisation Deleuzienne. Rien n'étant laissé au hasard, le « grand frère » de Léna subit sous hypnose le traumatisme fondateur, le viol par un inconnu devenu après la Réunification un metteur en scène sadique mais 
très admiré, viol qui symbolise celui commis par la dictature du SED (WL p. 72-75), la violence exercée par le parti contre son peuple. On découvre seulement à la fin du roman que, dans l'état hypnotique où son inconscient aurait dû trouver une réponse à son problème, le jeune homme n'a pu que révéler l'inhibition de ses fonctions vitales. Car la dictature est à l'origine de la " peste émotionnelle » (Wilhelm Reich) entraînant la frigidité et l'impuissance. À tous les niveaux, l'État en voie de disparition est gangrené par cette peste, les uns transmettant la maladie, les autres la subissant: parmi les "pestiférés ", dans le sens que Reich donne à ce terme, on compterait à l'est les policiers, le SED et la Stasi, et à l'ouest les hommes d'affaires, les investisseurs, les banquiers etc. tandis que les victimes de cette peste se réfugient dans diverses névroses : la frigidité et l'impuissance de Léna et de son " grand frère ", l'hyperactivité sexuelle de Daniel Detjen, comme, à l'ouest, celle de Léo Lattke, les plus conscients se perdant dans une lutte improductive (Daniel Detjen, Jürgen Warthe) ou la pensée utopique du petit poète, les plus cyniques choisissant le double jeu, l'intrigue et la trahison comme Gisela Blank.

10 À l'ouest également, une paralysie étrange frappe les meilleurs éléments : Léo a perdu son talent; face à l'univers mystérieux de ce continent qu'il ignore, lui, l'occidental prétentieux, arrogant et imbu de son génie, éprouve pour la première fois de sa vie le blocage de l'écrivain, incapable d'écrire une seule ligne du reportage commandé par son journal, sans doute le Spiegel. Cette difficulté à trouver l'inspiration, cette absence de créativité sont-elles seulement le produit d'une trop grande ambition, l'incitant à refuser la médiocrité du sujet, ou correspondent-elles à une prise de conscience, au refus de rentrer dans le rang et de se soumettre aux lois implacables du journalisme commercial?

11 Les structures du pouvoir conditionnent les structures psychologiques. Léo, Léna, le "Grand Frère ", tous incarnent les structures intériorisées de leur système politique : Léo, l'ambition sociale et économique, Léna et son grand frère, la castration par la dictature. Jeune, jolie, douée et exerçant une grande fascination sur ses concitoyens, Léna est pourtant, au fond d'elle-même, tout comme son double, celui qu'elle réduit au rôle de "grand frère" pour mieux freiner ses élans amoureux, une éternelle adolescente, l'enfant qui refuse de devenir femme.

12 Comme dans Helden wie wir, la révolution politique va de pair avec la découverte du corps, de la sexualité libre et sauvage. La chute du Mur constitue le choc salutaire seul capable de renverser les structures aliénantes et de permettre à l'individu de retrouver ses facultés pour jouir enfin de l'existence. Léna surmonte enfin sa frigidité; c'est seulement l'expérience cathartique du 9 novembre qui détruit en elle les blocages de la dictature. Léo ne renonce certes pas à ses ambitions, mais le séducteur insensible découvre enfin auprès d'elle une passion jusque-là ignorée. Avec la fin de la guerre froide, le dégel tant attendu depuis la mort de Staline, l'Allemagne sort d'une longue ère glaciaire.

13 La vague de fond de 1989 signifie la fin des habitudes, la fin des stéréotypes, mais aussi la fin des refoulements et des frustrations. Léo sent que sa rencontre avec Léna l'a radicalement transformé: grâce à sa nouvelle compagne, il retrouve sa naïveté juvénile, l'authenticité et la créativité perdue ${ }^{8}$. 


\section{Les automates}

14 Au ton larmoyant et immature de ses compatriotes, Brussig oppose la passion charnelle dont la violence seule peut détruire la raison castratrice transformant l'homme en un automate.

Le motif de l'homme-machine apparaît tout au long du roman. Lorsque Léna accompagne Léo à Hambourg, pour son premier séjour à l'ouest, elle assiste ainsi dans la rue à une performance de Breakdance au cours de laquelle un des danseurs se transforme en une poupée mécanique tandis que son partenaire est déjà un robot avant d'entrer en scène. L'homme-machine est finalement exterminé par la salve libératrice d'un fusil-mitrailleur qui, en l'exterminant, lui permet de retrouver la vie et de se relever souple, chaud et charnel...

Le danseur suivant ne se transforma pas en une chose, il arriva déjà à l'état de chose : comme un homme-machine visiblement de la première génération, car ses mouvements étaient saccadés et hachés, mécaniques et automatiques. Ses membres se détachaient avec raideur de son corps. Lorsqu'il se mouvait, tout semblait s'entrechoquer bruyamment. Et soudain, d'un instant à l'autre, il se transforma en un homme-machine de la deuxième génération, mû par une mécanique fluide et molle 9 .

Depuis le jour maudit de sa fuite à l'ouest, le père de Carola a cessé de croire en son pays, en lui-même, il vit comme un automate ${ }^{10}$. La fuite de sa fille en RFA, les événements de l'été et de l'automne 1989 ont fait vaciller ses certitudes. En perdant sa fille, il a perdu son âme. Seul son corps continue de servir mécaniquement un État auquel il ne croit pas.

18 Car la période qui précède la révolution de 1989 est décrite comme le temps du sommeil et de la léthargie. La RDA est un univers momifié, rigide et monolithique, si l'on en croit le roman de Waldemar inséré en abyme. Comme Leben bis Männer ${ }^{11}$, le sport y sert en effet de métaphore de la dictature. Si chez Waldemar, le sujet décrit le destin d'un entraîneur sportif momifié après s'être brisé plusieurs fois la nuque, l'auteur réel Brussig identifie l'entraîneur à un dictateur, un être médiocre et sans humanité, incarnation du petit bourgeois est-allemand soumis mais reproduisant les structures du pouvoir sur plus faible que lui.

19 La dictature a transformé les hommes en automates, en momies glacées; le 9 novembre leur a rendu la souplesse palpitante de la vie.

\section{Critique de la pensée utopique}

\section{Critique du Prenzlauer Berg}

20 Jürgen Warthe est certes un homme d'une grande droiture morale, un musicien de talent, mais il s'exprime sans passion, comme un automate lui aussi, ne parvenant pas à partager la joie de son peuple lorsque, le Mur ouvert par un miracle inouï ${ }^{12}$, les rues se transforment en un joyeux carnaval. Warthe, le dissident héroïque, paie de sa vie son opposition au SED, mais il a un défaut majeur : il est « ennuyeux " $^{13}$ ! Daniel Detjen, le dandy séducteur, l'intellectuel engagé du Prenzlauer Berg qui ne craint pas d'organiser des lectures “inofficielles ${ }^{14}$ dans son appartement, se disperse en d'inutiles correspondances avec le Nicaragua, les Indes, le Pakistan, la Palestine, les USA et même 
le Danemark; il se perd en d'improductives discussions sur la création d'une maison d'édition alternative; pourtant, la nuit du 9 novembre, alors que tout Berlin est en liesse, il ne trouve pas utile d'aller voir le prodige à deux pas de chez lui ${ }^{15}$. Avec Daniel Detjen, Jürgen Warthe et Waldemar, Brussig évoque la fameuse «Prenzlauer Berg Connection ", réunie autour de Jan Faktor et de Bert Papenfuß, lesquels avaient créé un groupe de rock, pour des soirées littéraires illégales dans des appartements du Prenzlauer Berg, comme dans la cuisine de Ekkehard Maaß par exemple.

21 Ni Carola ni son père, tous deux intellectuels, n'ont participé à l'allégresse du 9 novembre. Seule la mère, une femme simple, sans prétentions, parlant un dialecte saxon insupportable aux oreilles bien éduquées de son entourage, retrouve l'enthousiasme naif de la jeunesse en cette nuit fantastique. Car la chute du Mur, comme la prise de la Bastille, est l'œuvre du peuple, non point celle des intellectuels qui voient leur utopie renversée : Léna, la jeune kinésithérapeute, son "grand frère ", photographe amateur, l'ambulancier « Willi le sauvage », qui ignore la différence entre sanft et zart, etc. tous sont des gens simples et sans ambitions révolutionnaires, ils chantent, créent, vivent et sont prêts aux actions les plus folles, comme celle de détourner une ambulance, pour participer à la liesse générale après la chute du Mur. En vérité, Brussig souligne le paradoxe de l'unification : les intellectuels qui l'ont rendue possible, ne la voulaient pas!

Qui ne reconnaît pas Gregor Gysi dans l'avocate des dissidents Gisela Blank ${ }^{16}$ ? Jürgen Fuchs et Rudolf Bahro ont servi de modèle pour la conception du personnage de Jürgen Warthe et on sait que Gregor Gysi avait assuré la défense de Bahro lorsque ce dernier avait été emprisonné par la Stasi après la publication à l'ouest de L'Alternative ${ }^{17}$. Victime elle aussi de la "peste émotionnelle », Gisela, surnommée Gisi (WL 398), ne manifeste guère de générosité lorsque, sortant de l'opéra, elle félicite pour sa bonne mine son ancien client rongé par la leucémie. Malgré son QI exceptionnellement élevé, ou peutêtre à cause de cela, elle avait été condamnée par la Stasi au rôle de marionnette lors d'un jeu télévisé pour enfant où elle faisait le notaire; et selon le principe du comique carnavalesque, elle choisit Notar ${ }^{18}$ comme nom de guerre, soit le pseudonyme de Gregor Gysi lorsqu'il était IM pour la Stasi. Comme Gysi, jouant sans aucun remord un double jeu, Gisela défend les dissidents avec succès tout en servant la Stasi.

Et si Helden wie wir avait jeté son dévolu sur la première génération des braves qui avaient construit le socialisme, Wie es leuchtet ironise, avec une plus grande courtoisie sans doute, sur la nouvelle génération de héros, celle des intellectuels ${ }^{19}$ qui ont mis fin au régime honni sans oser aller jusqu'au bout et rejeter le socialisme. Déjà dans Helden wie wir, Brussig avait fustigé la sagesse raisonnable d'Adam Radomski, le rebelle respectueux de la loi et de l'autorité militaire. Car c'est justement ce désir de bien faire qui offre un terrain propice à l'éclosion du terrorisme et de la dictature. Là où les intellectuels mettaient la vertu, la justice - «Nous voulions la justice et avons obtenu l'État de droit ! $\aleph^{20}$, dit Bärbel Bohley après la signature du Traité d'Unification -, la morale, la réalisation de soi, Brussig met le bonheur et le plaisir, même aveugles, même contraires à la vertu et à la sagesse.

Sous le masque de la comédie burlesque, Thomas Brussig règle ses comptes avec le socialisme, la troisième voie des intellectuels incapables de renoncer à leur rêve, l'utopie d'un monde meilleur, tout comme avec les illusions de l'adolescence, pour accepter la nouvelle réalité à laquelle il faut faire face, cet occident qu'il faut apprendre à aimer quels que soient ses dangers et ses défauts. 


\section{La génération des anciens} services du SED ! Au lieu des membres du parti, réunis avec leurs familles, il ne trouve plus que des rochers, du sable, le mugissement des vagues sous un ciel gris. Tous les anciens serviteurs du socialisme s'agglutinent désormais sur le sable brûlant des Baléares ou de l'occidentale et bourgeoise Sylt. Le poète, présenté comme un membre privilégié de ce socialisme non égalitaire, déplore l'intégration de la RDA dans la RFA, en intervertissant les termes de la citation empruntée à Georg Büchner :

Krieg den Hütten, Friede den Palästen!

Tout comme Brussig avait retranscrit le discours de Christa Wolf du 4 novembre 1989 sur l'Alexanderplatz dans Helden wie wir, il cite intégralement le poème désabusé de Volker Braun dans Wie es leuchtet :

Da bin ich noch: mein Land geht in den Westen.

KRIEG DEN HÜTTEN FRIEDE DEN PALÄSTEN.

Ich selber habe ihm den Tritt versetzt.

Es wirft sich weg und seine magre Zierde.

Dem Winter folgt der Sommer der Begierde.

Und ich kann bleiben wo der Pfeffer wächst.

Und unverständlich wird mein ganzer Text.

Was ich niemals besaß wird mir entrissen.

Was ich nicht lebte, werd ich ewig missen.

Die Hoffnung lag im Weg wie eine Falle.

Mein Eigentum, jetzt habt ihrs auf der Kralle.

Wann sag ich wieder mein und meine alle $^{21}$.

des abus de la dictature, ne peuvent pas renoncer à leur rêve.

Le personnage représente tous les intellectuels qui, bien que très critiques envers le régime, n'ont pas opté pour la dissidence et n'ont pas su s'adapter à la nouvelle réalité politique. Volker Braun faisait partie, comme Christa Wolf, des écrivains est-allemands qui avaient jeté un regard sans concession sur le socialisme réellement existant. Mais cette objectivité critique ne l'avait point détaché de l'utopie socialiste que sa critique voulait réformer sans l'anéantir. Brussig force le trait et ne souhaite en rien décrire le véritable Volker Braun malgré l'insertion du poème. Il respecte sa droiture morale, reconnaît que ce que le poète regrette, ce n'est point le stalinisme, mais l'utopie, le rêve jamais réalisé d'un socialisme à visage humain. Il regrette seulement son incompréhension de l'accélération de l'Histoire. Brussig dit lui-même respecter le talent et la position inconfortable de Volker Braun en RDA.

Selon la tradition carnavalesque qui lui est chère, et sans tenir compte de la réalité, mais pour au contraire souligner que son personnage ne doit en aucun cas être identifié à Volker Braun ${ }^{22}$, notre auteur s'amuse à marier le petit poète nostalgique à un professeur de russe reconverti dans la privatisation de l'industrie pharmaceutique esteuropéenne. Là aussi la volonté blasphématoire transparaît ouvertement, les professeurs de russe étant d'habitude de fidèles serviteurs de l'Union soviétique. Si le poète, dans un élan de sincérité, critique l'impérialisme occidental, sa femme, pur produit de l'imagination de l'auteur, n'hésite pas par opportunisme à l'adopter ! Comme le héros de Jens Sparschuh dans Fontaine d'appartement, l'épouse s'adapte plus 
facilement au nouveau régime, un fait souvent observé par les sociologues, les hommes ayant éprouvé de plus grandes difficultés que les femmes à accepter les changements.

Comme Friedrich Dürrenmatt ${ }^{23}$, Brussig trouve dans le grotesque ${ }^{24}$, mélange saugrenu de réel et d'imaginaire, de tragique et de comique, le moyen le plus précis d'exprimer la réalité humaine et politique. Le grotesque exprime bien, comme Wolfgang Kayser ${ }^{25}$ l'a constaté, le changement des valeurs et des catégories, leur échec à expliquer le monde. Tout est possible, la raison ne suffit plus à expliquer le monde. Le grotesque ici correspond tout d'abord à la coexistence des contraires : le poète utopiste se voit marié à une femme d'affaires qui n'hésite pas à trahir sa foi socialiste pour adopter le capitalisme; le dissident habillé en femme est également grotesque, le communiste à l'œil de verre, l'albinos aux yeux rouges sont grotesques, les transsexuels, ni hommes ni femmes, en quête d'un chirurgien, le leur ayant fui à l'ouest sans terminer leur métamorphose, sont grotesques. La chute du Mur, en détruisant les normes "rationnelles, a plongé l'homme désorienté dans un univers absurde et multiple. Chez Brussig, le grotesque n'est pas, comme chez Dürrenmatt, une forme nouvelle du tragique, c'est plutôt le seul moyen de surmonter le tragique et d'appréhender la réalité du monde lorsque la pensée utopique a été écartée. Le poète qui, sans être dissident, ne jouit pas de la confiance absolue du parti qui censure la plupart de ses œuvres, est comparé à une araignée en train de pondre... et ses pensées forment une masse gluante... On sent que l'auteur réel partage l'aversion de son personnage Waldemar, un dissident polonais, pour la littérature est-allemande ${ }^{26}$. Mais on aurait tort d'attribuer la répugnance du personnage à son auteur. Si l'auteur ne juge pas utile de donner un nom à ce personnage désigné uniquement par sa fonction, c'est sans doute parce qu'il a un caractère paradigmatique: il incarne tous les écrivains qui, tout en critiquant le socialisme réellement existant, sont incapables de renoncer à leur utopie.

\section{Les communistes de la première heure}

Un autre personnage, lui aussi à la fois tragique et comique, victime du stalinisme, le vieux militant communiste Fritz Bode, a perdu un œil dans les prisons de la Stasi. Avec Fritz Bode, Brussig rappelle les procès staliniens qui, dans les années 1950, avaient permis à Walter Ulbricht d'éliminer des concurrents gênants tels que Kurt Müller, Leo Bauer, Willy Kreikemeyer, Bruno Goldhammer ou encore Franz Dahlem. En 1956, Wolfgang Harich, Walter Janka, Bernhard Steinberger, Gustav Just et Heinz Zöger sont arrêtés dans le cadre du soulèvement en Hongrie. Selon sa stratégie habituelle, Brussig dresse un portrait politique fidèle tout en inventant de nombreux détails biographiques qui donnent un caractère plutôt cocasse au personnage. Plusieurs éléments rappellent la biographie de Wolfgang Harich: Bode est condamné comme responsable du « complot hongrois » (WL 204); il travaille pour la SMAD, il se marie plusieurs fois..., se montre lâche devant la Stasi..., mais surtout, il ne renonce jamais à l'utopie socialiste ; aucune souffrance, aucune injustice ne peuvent lui ouvrir les yeux. Symboliquement, l'auteur lui en a d'ailleurs retiré un, signalant au lecteur que cet aveuglement n'est que partiel. L'ouverture du Mur, en lui rendant une importance perdue en RDA, lui permet de comprendre qu'il s'était fourvoyé toute sa vie ${ }^{27}$.

Comme il l'a déjà manifesté dans son roman Helden wie wir, Brussig se méfie des héros qu'ils soient socialistes ou non! Il leur préfère les petites gens qui construisent l'histoire à l'ombre du quotidien. Il hait les bonnes intentions, le désir de bien faire, la 
vertu toujours sûre de son droit, la volonté de créer un monde meilleur, l'utopie trop souvent socialiste et toujours totalitaire.

\section{Léonce et Léna ou l'union des contraires}

\section{Léonce et Léna}

Prisonniers d'un univers d'automates, de personnages clownesques, grotesques, corrompus, avides de richesses, et prêts à tout pour s'approprier les malheureux débris de la RDA moribonde, privés de leur énergie vitale, les personnages de Léo et de Léna ressemblent étrangement à ceux de Büchner, Léonce et Léna, ces jeunes gens désabusés en proie au dégoût de vivre après les désillusions de l'idéalisme romantique ${ }^{28}$. Léonce et Léna de l'unification, les deux personnages de Brussig, légers et chatoyants comme ceux de Georg Büchner, sont originaires de deux « royaumes », deux univers opposés. Le jeune prince Léonce doit épouser la jeune princesse Léna qu'il ne connaît point... Léna abhorre l'idée d'épouser un inconnu... Pour échapper à un destin odieux, ils prennent la fuite, chacun à l'insu de l'autre... Le journaliste Léo ignore tout de Léna et de la RDA qu'il méprise, Léna tout de Léo et de l'Ouest qu'elle craint. Longtemps, ils évoluent parallèlement, loin l'un de l'autre, se fuyant l'un l'autre...

Le journaliste adulé échoue dans son effort pour explorer ce continent inconnu, Léna, la rebelle, abhorre l'idée de disparaître avec sa nation si les deux États s'unissent. Or ce refus correspond à un manque de maturité, une prolongation artificielle de l'adolescence; Léna redoute l'entrée dans l'âge adulte et, avec elle, la remise en question de tout son être qui entraînerait la déstabilisation de sa personnalité.

Mais le hasard ou le destin faisant bien les choses, leurs chemins finissent par se croiser, non sans passer par une multitude de mésaventures entre rêve et réalité, pour provoquer le Happy end et l'union impossible ! Léo et Léna auraient-ils accepté l'inacceptable raison d'État pour rentrer dans le rang et rejoindre la place qui leur était assignée dans une Allemagne réunifiée ? Ou cherchent-ils leur bonheur hors de la voie imposée par l'Histoire ? Léna suit un itinéraire tumultueux et symboliquement fort qui, partant de Karl-Marx-Stadt, la mène à New York, tandis que Léo, le prince charmant de ce conte de fées postmoderne, quitte Hambourg pour sortir sa Belle au bois dormant de son sommeil maléfique et lui faire traverser l'Atlantique.

\section{Tom Wolfe et Bärbel Bohley : pas d'idéalisation de la RFA}

La RDA a disparu pour permettre la naissance d'une nouvelle entité qui ne doit pas seulement être l'addition de deux réalités ni l'absorption de la RDA par la RFA; car, grâce à la fusion des deux États -, symbolisée par l'union des deux contraires que sont le journaliste de style américain Léo, personnage inspiré par le populaire Tom Wolfe qui, mélangeant vécu, analyse et critique, est avec Hunter $\mathrm{S}$. Thomson un des inventeurs du nouveau journalisme, et Lena, surnommée Jeanne d'Arc, comme l'avait été dans la réalité historique Bärbel Bohley -, cette mort doit donner naissance à un être nouveau non encore défini... Certes, l'union de Tom Wolfe et de la vertueuse Bärbel Bohley produit un effet plutôt cocasse et relève du même esprit blasphématoire que la confusion de Christa Wolf avec Jutta Müller et Katharina Witt. Mais au-delà de la galéjade, on sent le désir de surmonter les oppositions et de réconcilier les deux États, 
en montrant comment les personnages les plus antithétiques peuvent s'associer, comment la RDA et la RFA devront se lier pour recréer l'Allemagne.

Si les intellectuels du Prenzlauer Berg manquent le rendez-vous de l'Histoire en ce 9 novembre 1989, vue de l'Ouest l'ouverture du Mur serait plutôt une catastrophe car elle met fin à une autre utopie : celle de pouvoir échapper à soi-même, de pouvoir changer de personnalité. Si Carola ne parvient pas à ressentir la joie de ses concitoyens, c'est qu'elle se sent rattrapée par son passé, otage de son identité est-allemande. Au lieu de se précipiter à la porte de Brandebourg, elle s'inquiète de trouver une place de parking pour la voiture de sa mère. Elle vient de subir un changement d'identité radical, en renonçant à ses rêves de jeune fille, à l'indolence orientale de la République démocratique, pour s'adapter à la rationalité et à l'efficacité occidentale. Que la liberté tant désirée prenne à l'Ouest les traits peu attrayants de la bureaucratie lui importe peu. L'Ouest signifie la fin des chimères, l'entrée dans l'âge adulte ; le temps des utopies fait place à l'esprit froid et calculateur (WL 105-106). L'aveugle qui avait développé une extrême sensibilité en RDA la perd dès que la chirurgie occidentale lui rend l'usage de ses yeux. À l'est comme à l'ouest, la chute du Mur signifie l'entrée dans la réalité, la fin des rêves, la fin des chimères.

La simple adhésion de la RDA à la République fédérale n'offre pas la solution la meilleure. La RDA ne doit pas adopter le rationalisme économique au prix de son identité. Ayant à peine vaincu la dictature du SED, le peuple de RDA ne doit pas aliéner sa jeune liberté en se tournant vers Mammon. Les hommes-machines ne doivent pas entrer dans un nouveau moule, mais au contraire renaître à la vie, retrouver la souplesse et la vigueur de la jeunesse. Car l'unification n'est pas idéalisée par le roman ; elle a ses victimes: Waldemar et Willi meurent de façon plutôt grotesque. La République fédérale apporte de nouvelles contraintes : la toute-puissance de l'argent et la corruption économique remplacent la dictature du SED et la perversion de la Stasi. Les intrigues politiques offrent des opportunités nouvelles aux chevaliers d'industrie... Ambitieux, vaniteux, toujours à l'affût de la sensation, Léo Lattke n'incarne ni la modestie ni la vertu ni le journalisme de qualité. Et la lumière qui l'éblouit à la fin du roman n'est point celle de Goethe mais plutôt celle du moloch new-yorkais. En s'alliant à lui, Léna renonce à ses certitudes, à sa vérité, à l'absolu de la raison, pour entrer dans le relatif scintillant, inquiétant, toujours en mouvement... Elle renonce à l'identité bien définie qui l'emprisonnait dans le rôle clos et immuable du héros de la révolution ${ }^{29}$, pour en endosser une autre sans contours fixes, mais brillante et contradictoire, ouverte à l'inconnu. Le scintillement de New York vu d'avion symbolise un avenir radieux, non encore enfermé dans une idéologie. Car ce que Léna apprécie, ce n'est pas le rêve américain mais le chatoiement de ses lumières à l'horizon, ce que son "grand frère ", son double, son alter ego, cherche à l'ouest, ce sont les nuages. Car plus que la liberté elle-même, c'est le désir de la liberté qui importe.

\section{Le rêve hippie : comment un vertueux dissident découvre le chanvre}

39 Tom Wolfe n'est pas seulement l'inventeur d'une autre forme de journalisme associant la réalité à la littérature, il est aussi l'auteur de Acid test, où il raconte le voyage hallucinant des Merry Pranksters ("joyeux lurons») en 1964 à travers les États-Unis dans un bus peinturluré où l'on consomme de la drogue en abondance. Le dernier épisode du roman dépasse en effet celui de l'unification des deux Allemagnes pour 
renouer avec le mythe californien des années 1960 : l'expérimentation de la drogue, le voyage intérieur, la recherche de la sérénité, l'attrait de la sagesse orientale. À l'utopie socialiste qui, aux yeux de Brussig, a définitivement échoué, à la société de consommation et à la corruption du capitalisme occidental que proposent la RFA et les USA avec leur nouvelle cathédrale du World Trade Center, le roman oppose l'alternative hippie ; pourtant associé à la mort, ce contre-modèle semble transposable.

Léo et Léna fuient en Amérique, le continent de tous les possibles, Warthe en Thaïlande... Waldemar et Big Brother prennent eux aussi leur envol, l'un en sautant dans les airs pour s'écraser, l'autre en apprenant à piloter un avion... Certains échouent ; d'autres poursuivent leur quête...

41 Comme dans Wasserfarben ou Le complexe de Klaus, Brussig présente la RDA sous un jour extrêmement noir. C'est la Stasi qui fait l'objet de la critique la plus virulente. Le père de Klaus meurt d'un cancer des intestins, victime d'une maladie hautement symbolique si l'on se réfère à l'analyse de W. Reich; ici, la Stasi transmet intentionnellement cette maladie mortelle. Le dissident Jürgen Warthe contracte la leucémie lors de son séjour dans les prisons de la Stasi qui l'a exposé à des rayons radioactifs. Cet épisode est inspiré de la vie de Jürgen Fuchs, qui aurait selon certains contracté sa leucémie de cette façon-là. La preuve n'a cependant jamais pu être établie avec certitude.

Étrangement, c'est au moment de quitter ce monde que Jürgen Warthe découvre le goût de vivre, le rêve hippie, message d'amour et de paix, d'union avec la nature, d'acceptation de soi, grâce à la drogue. Le voyage de Jürgen Warthe en Thaillande correspond au dernier voyage vers l'au-delà. À la suite d'Aldous Huxley, de Jack Kerouac et d'Allen Ginsberg pour qui l'Orient, la méditation Zen et la libération sexuelle ouvrent les portes de la perception, le dissident socialiste explore les voies nouvelles de la création non plus de l'art mais du moi. Il meurt réconcilié avec lui-même, avec sa femme et avec la nature. Son âme, symbolisée par le papillon, s'envole au moment où son corps disparaît à jamais et, avec lui, l'utopie socialiste. Mais comme l'a prédit Léna, le seul bruissement de ses ailes peut provoquer des bouleversements inconcevables. Comme pour la génération de 1970, la musique et la drogue offrent une alternative à l'Homme unidimensionnel de Marcuse. Et c'est à Allen Ginsberg, fasciné lui-aussi par le bouddhisme, que l'auteur confie la critique de ce monde formatés ${ }^{30}$.

43 La mort de Jürgen Warthe rappelle le séjour de Rudolf Bahro durant l'été 1983 à Rajneeshpuram, dans la commune de Bhagwan Shree Rajneesh (Osho) dans l'Oregon aux USA. Si Bahro a pris la défense de Bhagwan et du Rajneeshpuram, dont il avait pourtant critiqué les structures autoritaires, c'est qu'il avait ressenti, comme dans son livre L'Alternative, la nécessité de se changer soi-même avant de pouvoir changer le monde, l'un étant la condition de l'autre.

\section{Conclusion}

Wie es leuchtet serait-il une romance amoureuse célébrant, avec l'alliance de Léo et de Léna, Léonce et Léna de l'unification, le mariage des deux États allemands? Ou prônerait-il, à la suite de la génération hippie, l'éclatement des frontières de la perception, non point par la science, comme dans le cas de Sabine Busse, mais par la drogue, pour atteindre le Nirvana oriental? Rien n'est moins sûr. Dans une scénographie baroque placée sous le signe de l'impossible adieu à l'adolescence, Brussig nous plonge dans l'univers carnavalesque d'une révolution à laquelle il ôte le ton 
pathétique larmoyant adopté par nombre de ses confrères, un univers où le dérisoire et le politiquement incorrect côtoient la poésie de la nature et la violence de la passion. Diablotins, lutins aux yeux rouges, pantins du communisme, histrions du capitalisme, marionnettes de la dissidence, personnages cocasses, bouffons et polichinelles défilent dans une immense fresque carnavalesque... Léo, Léna, Big Brother, tous sortent transformés de l'expérience extraordinaire de l'ouverture du Mur vécue comme un traumatisme libérateur. Sous le masque trompeur d'un gai vaudeville, Brussig dénonce la comédie du pouvoir, la mascarade des réalités économiques d'une unification certes joyeuse mais non exempte de points noirs ni d'interrogations angoissées.

\section{BIBLIOGRAPHIE}

Grub, Frank Thomas : Wende und Einheit im Spiegel der Literatur. Ein Handbuch. 2 Bände, Berlin, New York, 2003.

Quéval, Marie-Hélène, «Irrungen und Wirrungen der Wende : Herr Lehmann, Sven Regener und Leander Haußmann » in : Volker Wehdeking (hg.) : Medienkonstellationen, Literatur und Film im Kontext von Moderne und Postmoderne, Marburg, Tectum Verlag, 2008.

Quéval, Marie-Hélène, « Le roman de Wolfgang Hilbig, "moi”, ou la déconstruction du moi ». Actes du colloques : L'impersonnel, Le Mans 2007, dir. Hélène Aji, Rennes, PUR, 2009.

Quéval, Marie-Hélène, L’Unification allemande, les années décisives 1989-1990, Éditions du Temps, Paris, 1998.

Quéval, Marie-Hélène, Die Aula d'Hermann Kant, Éditions du Temps, Paris, 1999.

Quéval, Marie-Hélène, dir., Günter Kunert, Éditions du Temps, Paris, 2000, ouvrage collectif avec une préface de Günter Kunert

Quéval, Marie-Hélène, dir., Ein weites Feld de Günter Grass, avec un texte inédit de H. J. Schädlich :

« Tallhover - Ein weites Feld », Paris, éd. du Temps, nov. 2001.

Wehdeking, Volker, Generationenwechsel : Intermedialität in der deutschen Gegenwartsliteratur, Erich Schmidt Verlag, Berlin, 2006.

\section{NOTES}

1. "Ein starrer, monolithischer Staat war wie pulverisiert.", Thomas Brussig, Wie es leuchtet, Frankfurt am Main. S. Fischer 2004 (sigle WL) p. 134.

2. « Frau Warthe warf Erde ins Grab, und als sie den Blick hob, sah sie einen Schmetterling, der, veranlaßt von ihrer Bewegung, seinen Flug begann. », WL p. 607.

3. En 1972, le météorologue Lorenz fait une conférence à l'American Association for the Advancement of Science intitulée: "Prédictibilité: le battement d'ailes d'un papillon au Brésil peut-il provoquer une tornade au Texas?» [ Predictability: Does the Flap of a Butterfly's Wings in Brazil Set off a Tornado in Texas?»]. 
4. «Alles ist Chaos! Sie warf die Hände in die Luft, um zu zeigen, was sie mit alles meinte. Sie war noch immer außer Atem und mußte ein paarmal tief Luft holen, ehe sie weiterreden konnte. „Die Chaostheorie sagt, daß der Flügelschlag eines Schmetterlings in Thailand etwas in Gang setzen kann, was zu einem Hurrikan in den USA führt. » WL, p. 53 «Darüber freu ich mich auch. Weil das nämlich bedeutet, daß der ganze Zauber hier auch mal zu Ende gehen kann.“» WL p. 54 « Überall hocken Zufälle. Das, was wir für den Zustand der Welt halten, ist wahrscheinlich etwas sehr Empfindliches, etwas Flüchtiges. Es beginnt immer mit einem Wassertröpfchen. Oder mit dem Flügelschlag eines Schmetterlings. Das ist mein Empfinden, mein Empfinden von der Welt. », WL, p. 55.

5. «Eine Schriftstellerin tritt aus der Partei raus. Ein Direktor lässt sich scheiden. Ein immer folgsamer Sportstar gibt andere Interviews. Ein Professor macht Yoga. Eine Tierärztin wird Vegetarierin. Ein Journalistikstudent bestellt die Zeitung ab. Ein Hausmeister hört auf zu rauchen. Eine Klavierlehrerin besucht einen Selbstverteidigungskurs. Eine Masseuse fährt auf Rollschuhen durch die Stadt. Alle machten etwas, das schon längst fällig war. Das Netz aus alten Gewohnheiten und Abhängigkeiten, aus Untätigkeit, Gleichgültigkeit und Ohnmacht war löchrig. Bald würde es ganz reißen. ", WL p. 58-59.

6. Félix Guattari, Chaosmose, Paris, Galilée, L'Espace Critique 1992.

7. Quéval, Marie-Hélène, Wenderoman, déconstruction du roman ou roman de la Déconstruction, le Roman en RDA (1985-1995), à paraître aux Presses universitaires de la Sorbonne, Paris, 2009.

8. «Leo Lattke fand, dass sie ein völlig anderer Mensch war als er. Nicht nur jünger, frischer, unverbrauchter und - er scheute sich dieses Wort zu denken - unverdorbener. Sie war inspirierend anders. Sie verstand auf ihre Art, was er schrieb. Er bekam eine Resonanz, die war originell und echt. ", WL p. 544.

9. «Der nächste Tänzer verwandelte sich erst gar nicht in ein Ding, er kam als Ding: Als ein Maschinenmensch, offenbar der ersten Generation, denn seine Bewegungen waren ruckhaft und abgehackt, von kantiger Mechanik. Die Gliedmaßen standen eckig vom Zentrum des Körpers ab. Alles an ihm schien zu scheppern, wenn er sich bewegte. Und plötzlich, von einem Moment auf den nächsten, verwandelte er sich in einen Maschinenmenschen der zweiten Generation, mit weicher fließender Mechanik. ", WL, p. 524.

10. «In der Nacht damals am Balaton, da ist was zerbrochen. Seitdem fühle ich mich wie ein Automat. » WL, p. 340.

11. Thomas Brussig, Leben bis Männer, Frankfurt am Main, S. Fischer, 2001

12. «Eine wirkliche Befreiung hatte stattgefunden. Es lag ein Leuchten über diesen Menschen, aus ihnen flutete rauschhafte Freude. Die Augen strahlten, die Mienen waren gelöst, ihre Bewegungen hatten etwas Spontanes, so daß auch Menschen über dreißig, ungeschickt wie Kinder, Hüpfer vollführten, sich gegenseitig anfaßten oder auf die Schultern klopften. Und überall dieses freudig-fassungslose Kopfschütteln.» WL, p. 135 . On reconnaît l'ironique référence au dicton des années 1968 : „Traue keinem über dreißig! ». En ce moment magique, même cette vérité-là devient caduque. La joie a un effet vivifiant, régénérateur, tout le monde retrouve l'enthousiasme de la jeunesse. Et comme dans l'hymne à la joie de Schiller et de Beethoven, tous les hommes sont frères: "Alle Menschen sind Brüder.» «Ein leuchtendes Staunen strahlte aus ihren Augen. », WL p. 97.

13. « Einen Jürgen Warthe zu interviewen, war das langweiligste von der Welt. », WL p. 134.

14. «Der kleine unrasierte Dichter war davon überzeugt, daß sich unter Ausschluß der Öffentlichkeit eine Öffentlichkeit entwickelt hatte, welche die stattliche, offiziell stattfindende Öffentlichkeit an Bedeutung übertroffen hatte. », WL p. 41.

15. «Er stellte das Radio an, um Nachrichten zu hören, doch es kamen keine. Als er aufgegessen hatte, ging er ins Wohnzimmer und sagte: «Die Mauer ist auf.» Barbara schaltete das Radio ein: Jubel an den Grenzübergängen, Trabis auf dem Ku'damm, Glücksgesänge am Brandenburger Tor. Lenas großer Bruder fotografierte die andächtig lauschende Schar. Ein leuchtendes Staunen floß 
aus den Augen. Er hatte noch nie Menschen knipsen können, die vom Eintreten des Utopischen überwältigt wurden. Die Reaktion war nicht Jubel, sondern feierliches Ergriffensein. », WL, p. 97. 16. Le terme Notar, surnom de l'IM Gregor Gysi lorsqu'il travaillait comme collaborateur inofficiel de la Stasi, apparaît en italique, ce qui lui confère un sens particulier. Or, c'est Gisela Blank qui accepte de jouer le rôle du Notaire lors d'une émission télévisée. WL p. 180.

17. Die Alternative. Zur Kritik des real existierenden Sozialismus, Köln/Frankfurt 1977.

18. «Sie sollte sich einen Decknamen wählen. Sie entschied mit bitterem Grinsen, sich Notar zu nennen. ", WL p. 182.

19. «In solchen Momenten werde ich von einer handfesten Intellektuellenfeindlichkeit heimgesucht. ", Helden wie wir, Frankfurt am Main 1998, p. 307.

20. «Wir haben Gerechtigkeit gewollt und haben den Rechtsstaat bekommen! », Bärbel Bohley, Ehrhart Neubert, Wir mischen uns ein, Ideen für eine gemeinsame Zukunft, Freiburg im Breisgau 1998, p. 84.

21. Die Zickzackbrücke. Ein Abrißkalender, Halle 1992, p. 84.

22. "Nur ganz schnell: Ich habe das Gedicht von Volker Braun genommen, weil ich es für sehr, sehr treffend halte. Ich kenne keinen anderen Text, weder von ihm oder jemand anderem, der auf so engem Raum das Dilemma des DDR-Intellektuellen nach der Wende (Identitätsproblematik) so gut beschreibt. / Volker Braun war im Gegensatz zu Christa Wolf keine Sockelfigur, und er hat danach auch nicht für sich in Anspruch genommen, im Widerstand gewesen zu sein. Er hatte nicht diese internationale Popularität, die ihn geschützt hätte, wenn er dissidentischer gewesen wäre. / Ich habe dem kleinen unrasierten Dichter eine Menge Dinge angehängt, die wirklich frei erfunden sind (seine Frau Russischlehrerin/dann Pharmazeutikavertreterin, alle Theaterstücke verboten bis auf eins, die Sache mit den Hörspielen, die nur einmal und dann nur um Mitternacht gesendet wurden und trotzdem im Underground zirkulierten, usw..) Nochmals: Volker Braun ist nicht gemeint, aber Volker Braun hat mir geholfen, diese Romanfigur zu entwerfen. Wer sich aber für Volker Braun interessiert, darf nicht auf die Idee kommen, deshalb die entsprechenden Passagen in «Wie es leuchtet» zu lesen. Dann gibt es nur Verwirrung. » Courriel à Marie-Hélène Quéval 26/07/08.

23. Philippe Wellnitz, Le théâtre de Friedrich Dürrenmatt. De la satire au grotesque, Strasbourg, Presses Universitaires de Strasbourg, 1999.

24. Isabelle Ost, Pierre Piret, Laurent Van Eynde, Le grotesque: Théorie, généalogie, figures, Publications des Fac. St Louis, 2004.

25. Wolfgang Kayser, Das Groteske, Seine Gestaltung in Malerei und Dichtung, Nachdruck der Ausgabe von 1957. Mit einem Nachwort „Zur Intermedialität des Grotesken“ von Günter Oesterle und mit einer aktuellen Auswahlbibliographie zum Grotesken, Monströsen und zur Karikatur. Stauffenburg Bibliothek, Band 1, 2004.

26. «Waldemar interessierte sich für das, was in diesem Lande passierte, und daß er aufhörte, sich für die Literatur dieses Landes $\mathrm{zu}$ interessieren, würde er den Schriftstellern nie verzeihen. ", WL p. 43.

27. «Fritz Bode hatte plötzlich das Gefühl, sein Leben in der falschen Branche verbracht zu haben. Nach einem kurzen Aufleuchten war er verglüht. », WL p. 210.

28. Beckers, Gustav, Georg Büchners „Leonce und Lena“, ein Lustspiel der Langeweile, Heidelberg: C. Winter, 1961.

29. «Das Jahrtausendhochwasser hat auch Lenas Lieblingsphotos vernichtet. Auf diesen Bildern war sie so „schön wie nie“, sagt sie, und es stimmt. Neunzehn war sie damals. Es war die aufregendste Zeit in ihrem Leben. Ihr Lied war Nummer eins in der Hitparade, und sie war eine Volksheldin. Ich war stolz darauf, sie zu kennen, und damals besonders. », WL, p. 12.

30. « Moloch dessen Geist reine Maschinerie ist! / Moloch dessen Blut als Geld fließt! / Moloch dessen Ohr ein rauschendes Grab ist! / Moloch mit tausend blinden Fenstern als Augen! » WL, p. 571. 


\section{RÉSUMÉS}

Le roman Wie es leuchtet de Thomas Brussig présente la situation de l'Allemagne unifiée en 2002 et les troubles identitaires provoqués par la chute du Mur en RDA. La perspective est positive, car, comme dans Le Complexe de Klaus (Helden wie wir), l'ouverture du Mur bouscule les structures figées de la société est-allemande. Reprenant la théorie de Lorenz sur l'effet du papillon, Brussig montre que des événements presque insignifiants, comme le fait qu'un bon bourgeois cesse de laver sa voiture le dimanche, peuvent annoncer des changements extraordinaires. Ainsi en va-t-il de chaque personnage dans ce roman. L'unification allemande créant un choc salutaire, on assiste à la transformation complète des caractères, à un changement radical des identités aussi bien à l'est qu'à l'ouest. Imprégné de la pensée antiautoritaire et libertaire des années 1970, Brussig part des analyses d'Alice Miller et de Wilhelm Reich, établissant un rapport entre la répression de la sexualité et la dictature. Le Mur, symbole de cette répression, libère, en disparaissant, les citoyens immatures de leur éternelle adolescence et leur permet d'entrer dans l'âge adulte. Enfin libres et responsables, ils affrontent, en passant de l'autre côté du Mur, le monde infini, insaisissable, avec toutes ses contradictions. L'image finale du papillon qui s'envole annonce d'autres bouleversements dans un univers enfin en mouvement perpétuel.

Brussigs Roman Wie es leuchtet spielt im Jahr 2002 und stellt die Wirren der Vereinigung aus der DDR-Perspektive dar. Ohne die Schwierigkeiten der Vereinigung zu leugnen, liefert Brussig eine optimistische Darstellung der Wendezeit. Denn wie in Helden wie wir lockert die Öffnung der Mauer die erstarrten Strukturen der DDR-Gesellschaft auf. Der Roman geht von Lorenz' Theorie des Schmetterlings aus, um zu zeigen, daß unscheinbare Änderungen im Leben eines Kleinbürgers, der z. B. sonntags sein großes Auto nicht mehr waschen will, große Umwälzungen bewirken können. Die deutsche Vereinigung provoziert einen gesunden schockartigen Bruch mit der Vergangenheit, eine radikale Veränderung der Charaktere und Identitäten sowohl im Westen als auch im Osten. Beeinflußt vom libertären und antiautoritären Gedankengut der 1970er Jahre, geht Brussig von Alice Millers und Wilhelm Reichs psychoanalytischen Interpretationen des National-Sozialismus aus, um den Zusammenhang zwischen Unterdrückung der Sexualität und Diktatur zu veranschaulichen. Mit dem Fall der Mauer als Symbol der Repression wird der bisher unmündige DDR-Bürger in das Erwachsenenalter mit all seinen Freiheiten und unendlichen, wenn auch kontradiktorischen Möglichkeiten entlassen. Das letzte Bild vom dahinfliegenden Schmetterling kündigt neue unendliche und unvorhersehbare Entwicklungen in einer sich ständig verändernden Welt an.

\section{AUTEURS}

\section{MARIE-HÉLÈNE QUÉVAL}

Université du Maine (Le Mans) 